\title{
Comparative Lipid Profiles of Milk Bank Breast Milk and Infant Formulas
}

\author{
Anya Oleynik, Tatiana Eliseeva and Jack Y. Vanderhoek*
}

Department of Biochemistry and Molecular Medicine, The George Washington University, School of Medicine and Health Sciences, Washington, DC 20037

\begin{abstract}
Lipid profiles of seven human breast milk samples obtained from milk banks and four infant formulas were compared in view of the potential food hypersensitivities of certain infants to human milk. The cholesterol $(0.15-0.26 \mathrm{mM})$ content of the human samples was about 50\% lower than that found in the infant formulas whereas the triglyceride (TG, 173-386 mM) contents of these products were found to be comparable. The major saturated fatty acid (SFA) and monounsaturated fatty acid (MUFA) were 16:0 and 18:1 respectively. The major poly-unsaturated fatty acid (PUFA) was 18:2 with other PUFA members of the C18, C20 and C22 families identified and quantified. Although conjugated linoleic acid (CLA) was not detected in any infant formulas tested, no other major differences in the fatty acid patterns were found. However, the mean (13.6) of the ratio of n-6 PUFAs/n-3 PUFAs in the human milk samples was about $50 \%$ higher than that observed in the infant formula samples. Although our results indicate that there are small yet significant differences in cholesterol and CLA content and the ratio of n-6 PUFAs/n-3PUFAs, the lipid composition of milk bank breast milk and infant formulas is quite comparable.
\end{abstract}

Keywords: cholesterol,CLA, fatty acids, human, milk, PUFA, triglycerides.

\section{INTRODUCTION}

Milk has long been an essential component in human nutrition. Newborns totally depend on "mothers' milk" for their sustenance and according to the World Health Organization, breast feeding for the first six months is advantageous to the infant's development and long-term health status [1]. However, under certain circumstances breast-feeding is either impractical or undesirable, e.g for premature infants, special needs infants or the mother's medical condition, and mothers who still want to provide human milk for their infants have turned to human milk banks since wet nurses are no longer in vogue.To prevent disease transmission, human milk banks screen donor milk for various diseases prior to distribution. Other mothers have opted for infant formulas to feed their babies as these commercial products are easily accessible and manufactured to conform to certain standards. Obviously each nutritional option (milk bank or commercial formula) represents different benefits and risks [2, 3].

Both human milk and infant formula contain milk triglycerides as the major source of energy in addition to other essential nutrients for infants. A number of studies have examined the lipid profiles of human milk samples [4-6] and several studies have compared the lipid composition of human milk and infant formulas [7-9]. In most of these studies, milk samples were collected at different post-partum stages and immediately frozen and stored prior to analyses.

"Address correspondence to this author at the Department of Biochemistry and Molecular Medicine, The George Washington University, School of Medicine and Health Sciences, Washington, DC 20037; Tel: 202-994-2929; Fax: 202-994-8974; E-mail: jyvdh@gwu.edu
However, milk banks only use mature breast milk and routinely pasteurize donor milk samples prior to distribution [2]. Since we are not aware of any reports concerning milk lipid analyses from samples obtained from milk banks, we carried out the present study and compared the lipid composition of pasteurized breast milk from human milk banks and several infant formulas in order to determine which milk group might be a better lipid source for infant nutrition.

\section{EXPERIMENTAL SECTION}

All infant formulas used in this study were purchased from local grocery stores. The infant formulas purchased were Enfamil LIPIL ${ }^{\circledR}$ infant formula, Nestle Good Start Gentle Plus ${ }^{\circledR}$ formula, Similac Advance Early shield ${ }^{\circledR}$ infant formula and SimilacIsomil Advance ${ }^{\circledR}$ soy formula. The human breast milk samples were obtained either from the Mothers' Milk Bank (Raleigh, NC) or the Breastfeeding Center of Greater Washington (DC). Approval for the use of human subjects was obtained from the Office of Human Research, The George Washington University, and informed consent was obtained from the donors.

Triglyceride (TG) analyses were carried out using the Triglyceride Infinity reagent TR 22321 (Thermo Fisher Scientific, Inc., Atlanta, GA) according to the protocol described in reagent technical bulletin. Cholesterol quantitation was determined using the fluorogenicAmplex Red cholesterol assay kit (A12216) obtained from Invitrogen (Carlsbad, CA) following the procedure described in the kit bulletin. GLC analyses were carried out with a helium carrier gas on a Supelcowax 10 fused silica capillary GLC column \# 24081 (60m x $0.25 \mathrm{~mm}$ ID, $0.25 \mu \mathrm{m}$, Supelco, Inc. Bellefonte, PA). 
The column was attached to a Shimadzu GC 14A chromatograph connected to a Shimadzu CR8A Chromatopac data processor (Shimadzu Scientific Instruments, Inc., Columbia, MD). In order to determine the fatty acid content of the milk samples, the milk lipids were first extracted using the Folch extraction method [10] and then transmethylated using the sodium methoxide approach [11]. The fatty acid methyl esters (FAMEs)were analyzed using injector and flame ionization detector temperatures set at $250^{\circ} \mathrm{C}$. Two different column temperature programs were run for each sample: program 1 started at $100^{\circ} \mathrm{C}$ for $1 \mathrm{~min}$, then $100^{\circ}$ to $210^{\circ} \mathrm{C}(10$ $\mathrm{min}), 210^{\circ} \mathrm{C}(60 \mathrm{~min}), 210^{\circ}$ to $240^{\circ} \mathrm{C}(5 \mathrm{~min})$ and $240^{\circ} \mathrm{C}$ for 5 min; program 2 started at $65^{\circ} \mathrm{C}$, then $65^{\circ}$ to $195^{\circ} \mathrm{C}(10$ $\min ), 195^{\circ} \mathrm{C}(20 \mathrm{~min}), 195^{\circ}$ to $240^{\circ} \mathrm{C}(3 \mathrm{~min})$ and $240^{\circ} \mathrm{C}$ for 75 min [11]. A known amount of 13:0 methyl ester was used as an internal standard for all GLC analyses as separate experiments had shown the absence of this FAME in all original milk samples. GLC reference FAME standard mixtures \# 461, 538, 606 and some individual FAMEs were obtained from Nu-Chek Prep, Inc. (Elysian, MN) to confirm the identities of the FAME components in the milk samples. Each sample was analyzed at least twice to ensure reproducibility.

\section{RESULTS}

As shown in Table 1, the cholesterol content of seven human breast milk samples obtained from milk banks ranged from 0.155 to $0.265 \mathrm{mM}$ (mean $0.219 \mathrm{mM}$ ) whereas the cholesterol content of the infant formulas was about $50 \%$ higher $(\mathrm{p}<0.05)$ and ranged from $0.250-0.385 \mathrm{mM}$ (mean 0.318 $\mathrm{mM}$ ). The TG concentrations of the seven human donors varied from 173 to $386 \mathrm{mM}$ (mean $292 \mathrm{mM}$ ) which was comparable to the TG content range of the infant formulas, 228-297 mM (mean $274 \mathrm{mM}$ ).
The fatty acid profiles of human breast milk samples from seven donors and four different baby formulas are presented in Tables $\mathbf{2}$ and 3. In general, a number of qualitative similarities between these groups were observed. For example, the major saturated fatty acid (SFA) and monounsaturated fatty acid (MUFA) are palmitic acid and octadecenoic acids respectively. In human breast milk, the palmitic acid content ranged from 18.4-28.0 mole \% (mean $22.8 \%$ ) and in infant formula from 8.81-24.4\%. The octadecenoic acid content in human breast milk ranged from $35.4-50.7 \%$ (median $40.5 \%$ ) and in baby formulas from $37.0-46.1 \%$. The major poly-unsaturated fatty acid (PUFA) present in all these samples is linoleic acid with lesser amounts of 18:3(n-3). In the human donors, the linoleic acid content ranged from 14.2-22.1 \% (mean $18.0 \%$ ) and in infant formulas, the range was from $17.6-23.7 \%$. The PUFA family with the highest mole \% was the $\mathrm{C} 18$ and included 18:2 (n-6), 18:3 (n-6), 18:3 (n-3) and conjugated linoleic acid (CLA). In human breast milk samples, the total C18 PUFA mole \% ranged from 16.1-24.4\% (mean 19.6\%) and in infant formula, from $19.3-25.9 \%$ (Table 4). For all milk samples, the relative mole \% content for the three PUFA families was in the order of $\mathrm{C} 18>>\mathrm{C} 20>\mathrm{C} 22$ (Table 4). No CLA isomers were detected in any of the infant formulas.

When the ratios of (SFA+MUFA)/PUFA were determined, the human milk samples [ratios ranged from 2.784.64 (mean 3.78)] were comparable to those calculated for the infant formula samples [ratios varied from 2.72-3.84 (mean 3.15), Table 4]. The ratio of $n-6$ PUFA/n-3 PUFA is generally utilized to determine the balance between essential fatty acids. As shown in Table 4, this ratio varied from 7.6320.1 for seven human donors and from 9.00-9.64 for infant formula samples. The mean (13.6) of this $n-6 / n-3$ ratio for human donors was significantly higher $(\mathrm{p}<0.05)$ than the corresponding mean (9.35) observed for the formula samples.

Table 1. Cholesterol and TG Levels in Milk Samples from Human Breast and Infant Formulas. Cholesterol and TG Analyses were Carried Out as Described in Materials and Methods. Data are mean \pm SD (n) where $n$ is the number of analyses Carried Out

\begin{tabular}{|c|c|c|}
\hline Source & Cholesterol (mM) & Triglycerides (mM) \\
\hline \multicolumn{3}{|c|}{ Human } \\
\hline Subject 1 & $0.252 \pm 0.037$ & $364 \pm 80(4)$ \\
\hline Subject 2 & $0.235 \pm 0.035$ & $273 \pm 72(4)$ \\
\hline Subject 3 & $0.215 \pm 0.025$ & $278 \pm 66(4)$ \\
\hline Subject 4 & $0.192 \pm 0.038(2)$ & $173 \pm 10(4)$ \\
\hline Subject 5 & $0.265 \pm 0.045(2)$ & $386 \pm 73(4)$ \\
\hline Subject 6 & $0.217 \pm 0.052(2)$ & $275 \pm 37(4)$ \\
\hline Subject 7 & $0.155 \pm 0.005$ (2) & \\
\hline \multicolumn{3}{|c|}{ Infant Formula } \\
\hline Enfamil & $0.250 \pm 0.050$ & $278 \pm 13(4)$ \\
\hline Goodstart & $0.337 \pm 0.023$ & $291 \pm 13(4)$ \\
\hline Similac & $0.300 \pm 0.050(2)$ & $228 \pm 44(4)$ \\
\hline Simlc/Isomil & $0.385 \pm 0.045(2)$ & $297 \pm 15(4)$ \\
\hline
\end{tabular}


Table 2. Fatty Acid Composition of Lipids Extracted from Human Breast Milk. After Extraction, Lipids were Trans-esterified to Fatty Acid Methyl Esters and Analyzed by Capillary GLC as Described in Materials and Methods. Data are Expressed as Mole \% and are the Average of at Least two Separate Determinations

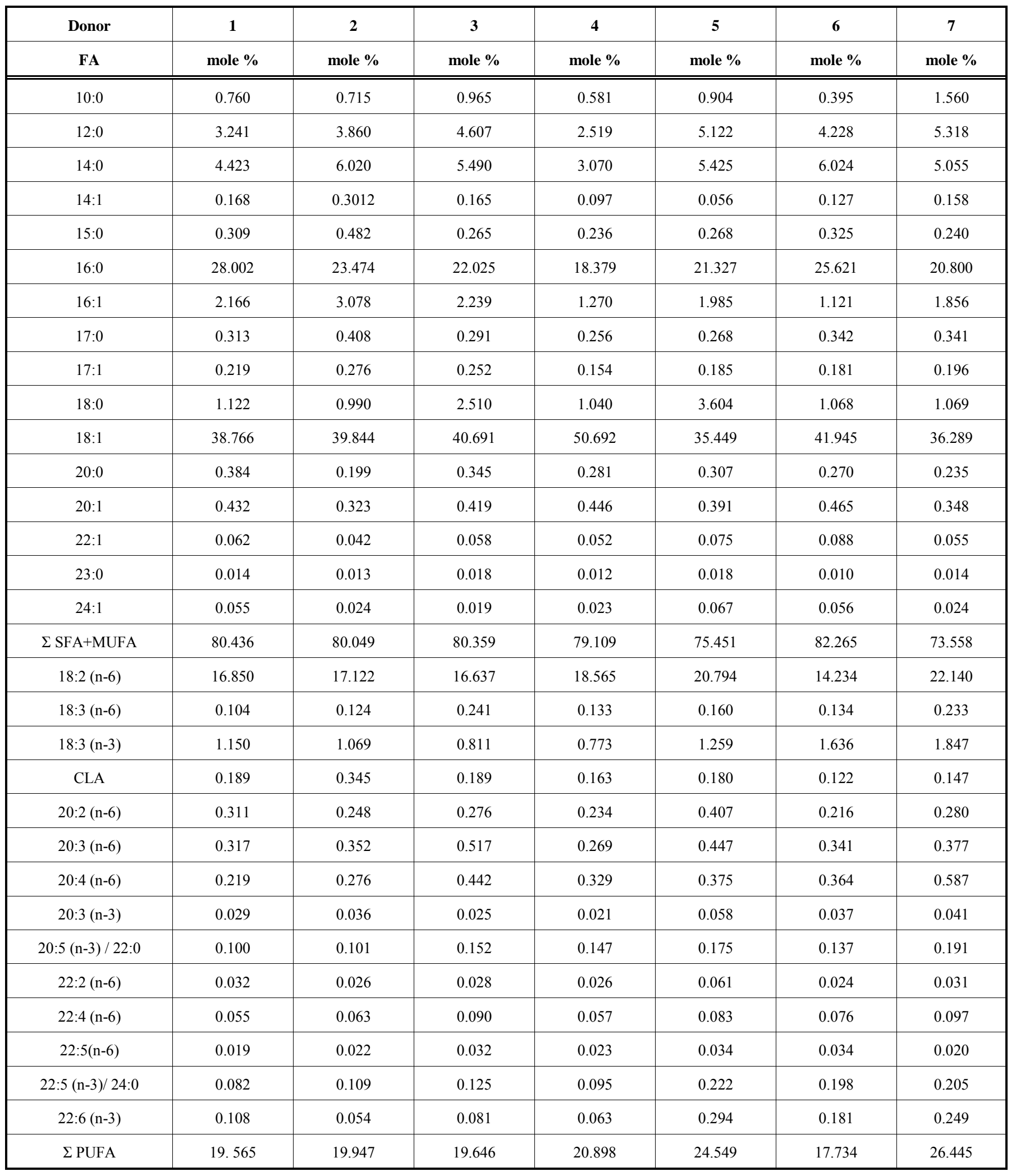


Table 3. Fatty Acid Composition of Lipids Extracted from Several Infant Formula Samples. See Legend to Table 2 for Explanation. ${ }^{1}$ Not Detected

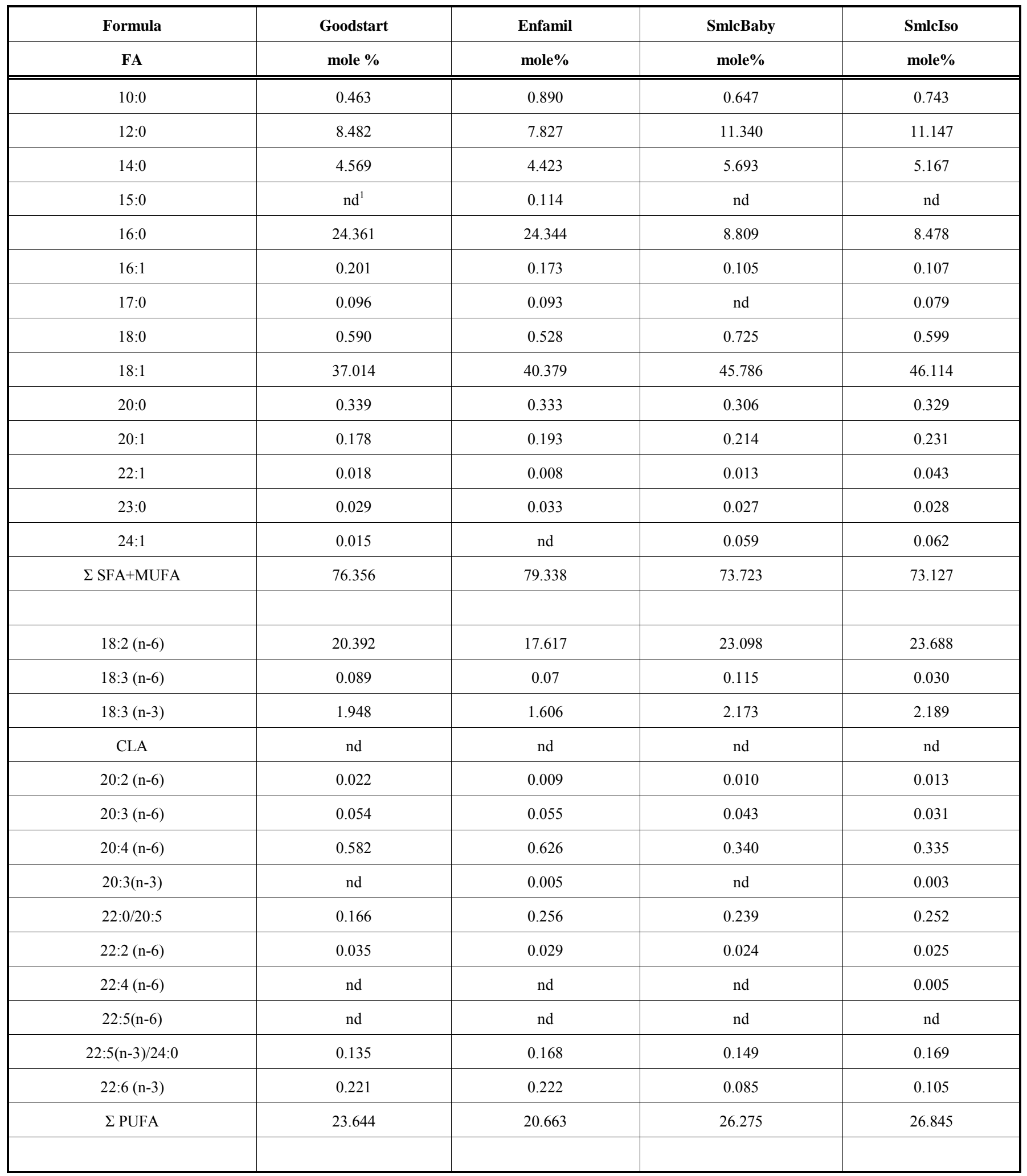


Table 4. Comparison of Fatty Acid ratios from Human Milk and Infant Formula Sources. ${ }^{2}$ These Calculations are Based on the Assumption that the GLC Peaks Representing Mixtures of 20:5 (n-3) + 22:0 and of 22:5 (n-3) + 24:0 Consist of Equal Amounts of Each Component

a. Human Milk

\begin{tabular}{|c|c|c|c|c|c|c|c|}
\hline Donor & 1 & 2 & 3 & 4 & 5 & 6 & 7 \\
\hline$\Sigma$ C18 PUFA & 18.293 & 18.660 & 17.878 & 19.634 & 22.393 & 16.126 & 24.367 \\
\hline$\Sigma \mathrm{C} 20$ PUFA & 0.926 & 0.963 & 1.336 & 0.927 & 1.375 & 1.027 & 1.381 \\
\hline ratio $(\mathrm{SFA}+\mathrm{MUFA})^{2} / \mathrm{PUFA}$ & 4.11 & 4.01 & 4.09 & 3.79 & 3.07 & 4.64 & 2.78 \\
\hline
\end{tabular}

b. Infant Formula

\begin{tabular}{|c|c|c|c|c|}
\hline Formula & Goodstart & Enfamil & SmlcBaby & SmlcIso \\
\hline$\Sigma$ C18 PUFA & 22.429 & 19.293 & 25.386 & 25.907 \\
\hline$\Sigma$ C20 PUFA & 0.741 & 0.823 & 0.512 & 0.508 \\
\hline ratio $\left(\mathrm{SFA}+\mathrm{MUFA}^{2} / \mathrm{PUFA}\right.$ & 3.23 & 3.84 & 2.81 & 2.72 \\
\hline
\end{tabular}

\section{DISCUSSION}

This study compared the lipid profiles of seven milk samples obtained from American milk banks with four types of infant formulas with regard to cholesterol and triglyceride levels and fatty acid composition. The TG content of the human milk and infant formulas were comparable but the cholesterol content of the latter was about $50 \%$ higher than that found for the human samples. Our human cholesterol milk data is about half that reported by Huisman et al. [8] which may be a reflection of the different diets consumed by American and Dutch women and/or a change in eating habits between these groups over the last 17 years.
The fatty acid profiles of our human milk samples were quite similar to those reported for European women $[8,9$, 12] and it seems unlikely that the typical pasteurization process used by milk banks [2] has any influence on the lipid profile of breast milk samples. In addition, the fatty acid composition of the American infant formulas tested in this study were comparable to those reported for Dutch, German and Spanish commercial infant formulas [8, 9, 13]. In contrast to our human PUFA profiles, no CLA [a family of isomers with beneficial effects $[14,15]$ was detected in our infant formula products. However, since many dairy and meat products contain CLA [16], once the infant is weaned from infant formula, these known CLA-containing foods are 
common dietary components. A similar concern for the absence of 22:4 (n-6), an essential precursor to very long-chain (n-6) PUFAs needed for normal spermatogenesis and fertility [17], in any of the infant formulas may be forestalled in view of the presence of 20:4 (n-6), the precursor to 22:4 (n6).A number of reports have shown that both 20:4 (n-6) and 22:6 (n-3) levels in human milk correlate positively with infant growth and development $[18,19]$. Our data indicate that the 20:4 (n-6) and 22:6 (n-3) mole \% ranges for the human samples (0.22-0.59 and 0.054-0.29 respectively) are quite comparable to those found for the infant formulas (0.34-0.63 and 0.085-0.22 respectively).

n-6 PUFAs and n-3 PUFAs are precursors to different oxidized metabolites (e.g. eicosanoids) that tend to have opposing biological effects in such diverse activities as cellular aggregation, immunological and inflammatory processes [20]. Consequently, the $n-6$ PUFA/n-3 PUFA ratio (n6/n3) has usually been used as a marker to indicate which of these metabolites are expected to be dominant [21]. Our studies indicate that this $\mathrm{n} 6 / \mathrm{n} 3$ ratio for the human milk (mean 13.6) was significantly (about 50\%) higher than that found for the infant formulas.

\section{CONCLUSION}

Despite the fact that the World Health Organization recommends that mothers breastfeed their infants for the first 6 months, certain circumstances may make the mother's own milk unavailable. Alternative choices are breast milk from milk banks or commercial formula though each option represents different benefits and risks $[2,3]$. Our small study indicates that there are small, yet significant, differences in the lipid composition (i.e. cholesterol and CLA contents and certain fatty acid profiles) of milk bank breast milk and infant formulas but that these differences are not sufficient to prioritize either nutritional source.

\section{ABBREVIATIONS}

$$
\begin{array}{ll}
\text { CLA } & =\text { Conjugated linoleic acid } \\
\text { FAME } & =\text { Fatty acid methyl ester } \\
\text { MUFA } & =\text { Mono-unsaturated fatty acid } \\
\text { PUFA } & =\text { Poly-unsaturated fatty acid } \\
\text { SFA } & =\text { Saturated fatty acid } \\
\text { TG } & =\text { Triglyceride }
\end{array}
$$

\section{ACKNOWLEDGMENT}

A.O. was the recipient of a 2009-10 Luther Rice Undergraduate Fellowship from the Columbian College of Arts and Sciences of The George Washington University.

\section{REFERENCES}

[1] www.who.int/mediacentre/factsheets/fs342 (cited 2013, October 21).

[2] Kim JH, Unger S. Human milk banking. Paediatr Child Health 2010; 15(9): 595-8.

[3] Gribble KD, Hausman BL. Milk sharing and formula feeding: infant feeding risks in comparative perspective? Aust Med J 2012; 5(5): 275-283.

[4] de la Presa-Owens S, Lopez-Sabater MC, Rivero-Urgell M. Fatty acid composition of human milk in Spain. J Ped Gastroenter Nutr 1996; 22(2): 180-5.

[5] Szabo E, Boehm G, Beermann C, et al. Trans octadecenoic acid and trans octadecadienoic acid are inversely related to long-chain polyunsaturates in human milk: results of a large birth cohort study. Am. J Clin Nutr 2007; 85: 1320-6.

[6] Wu TC, Lau BH, Chen PH, Wu LT, Tang RB. Fatty acid composition of Taiwanese human milk. J Chin Med Assoc 2010; 73(11): 581-8.

[7] Jensen RG, Ferris AM, Lammi-Keefe CJ. Lipids in human milk and infant formulas. Annu Rev Nutr 1992; 12: 417-41.

[8] Huisman M, van Beusekom CM, Lanting CI, Nijeboer HJ, Muskiet FAJ, Boersma ER. Triglycerides, fatty acids, sterols, mono- and disaccharides and sugar alcohols in human milk and current types of infant formula milk. Eur J Clin Nutr 1996; 50: 255-60.

[9] Lopez-Lopez A, Lopez-Sabater MC, Campoy-Folgoso C, RiveroUrgell M, Castellote-Bargallo AI. Fatty acid and sn-2 fatty acid composition in human milk from Granada (Spain) and in infant formulas. Eur J Clin Nutr 2002; 56: 1242-54.

[10] Folch J, Lees M, Stanley GHS. A simple method for the isolation and purification of total lipids from animal tissues. J Biol Chem 1957; 226: 497-509.

[11] Kramer JKG, Blackadar CB, Zhou J. Evaluation of two GC columns (60 m Supelcowax 10 and $100 \mathrm{~m}$ CP Sil 88) for analysis of milk fat with emphasis on CLA, 18:1, 18:2 and 18:3 isomers, and short- and long-chain FA. Lipids 2002; 37(8): 823-35.

[12] Koletzko B, Mrotzek M, Eng B, Bremer HJ.Fatty acid composition of mature human milk in Germany. Am J Clin Nutr 1988; 47: 9549.

[13] Koletzko B, Bremer HJ. Fat content and fatty acid composition of infant formulas. Acta Paedeatr Scand 1989; 78: 513-21.

[14] Banni S, Heys SD, Wahe KWJ. Conjugated linoleic acids as anticancer nutrients: studies in vivo and cellular mechanisms. In: Sebedio JL, Christie, WW, Adlof R, Eds. Adv. Conjug Linoleic Acid Research. Vol. 2. 2003; pp. 267-282.

[15] Cook ME, Butz D, Li G, Pariza M, Whigman L, Yang M. Conjug linoleic acid enhances immune responses but protects against the collateral damage of immune events. In: Sebedio JL, Christie, WW and Adlof R, Eds.Adv. Conjugated Linoleic Acid Research. Vol. 2. 2003; pp. 283-291.

[16] Parodi PW.Conjugated linoleic acid in food.In:Sebedio JL, Christie, WW and Adlof R, Eds.Adv. Conjug Linoleic Acid Research. Vol. 2. 2003; pp. 101-122.

[17] Zadravec D, Tvrdik P, Guillou H, et al. ELOVL2 controls the levels of n-6 28:5 and 30:5 fatty acids in testis, a prerequisite for male fertility and sperm maturation in mice. J Lipid Res 2011; 52: 245-55.

[18] Innis SM. Human milk: maternal dietary lipids and infant development. Proc Nutr Soc 2007; 66: 397-404.

[19] Agostoni C. LC-PUFA content in human milk: is it always optimal? Acta Paediatr 2005; 94: 1532-7.

[20] Serhan CN, Savill J. Resolution of inflammation: the beginning programs the end. Nat Immunol 2005; 6(12): 1191-7.

[21] Lands W. Consequences of essential fatty acids. Nutrients 2012; 4 : 1338-57. 